\title{
Robust Multisampled Capacitor Voltage Active Damping for Grid-Connected Power Converters
}

\author{
Javier Samanes $^{\mathrm{a}, *}$, Andoni Urtasun ${ }^{\mathrm{a}}$, Eugenio Gubia ${ }^{\mathrm{a}}$, Alberto Petri $^{\mathrm{b}}$ \\ ${ }^{a}$ Public University of Navarre, Campus Arrosadia, Pamplona, Spain \\ ${ }^{b}$ Ingeteam Power Technology, Av. Ciudad de la Innovacion, Sarriguren, Spain
}

\begin{abstract}
The derivative feedback of the capacitor voltage is one of the most extended active damping strategies, used to eliminate stability problems in grid-connected power converters with an $L C L$ filter. This strategy is equivalent to the implementation of a virtual impedance in parallel with the filter capacitor. This virtual impedance is strongly affected by the control loop delays and frequency, creating changes in the sign of the emulated virtual resistor, and raising instability regions where the active damping is ineffective. As a consequence, the $L C L$ resonance frequency is restricted to vary, as the effective grid inductance changes, within the active damping stability region. This is an additional restriction imposed on the $L C L$ filter design that can compromise the achievement of an optimised design. For this reason, in this work, a different strategy is presented; by adjusting the delay in the active damping feedback path, it becomes stable within the range where the $L C L$ resonance frequency can be located for a given filter design, achieving a robust damping. Analytical expressions are provided to adjust this delay. To widen the stability region of the capacitor voltage derivative active damping, a multisampled derivative is implemented, overcoming its limitations close to the control Nyquist frequency. Experimental and simulation results validate the active damping strategy presented.
\end{abstract}

Keywords: Active damping, capacitor voltage derivative, converter control, grid connected power converter, $L C L$-filter, multisampling.

\section{Introduction}

Grid-connected voltage source power converters (VSC) are largely used as an interface with the grid for renewable power systems [1, 2, 3, 4. As the number of power converters increases, stringent grid codes regulating the power quality injected have been developed [5]. A common approach to meet these grid codes 5 is the use of an $L C L$ filter, reducing its overall size and cost if compared with the $L$ filter $[\underline{6}$. In this filtering topology, the resonance between the filter and grid impedances must be damped, as stability issues arise

\footnotetext{
* Corresponding author

Email address: javier.samanes@unavarra.es (Javier Samanes)
} 
otherwise due to the effects of the delays in the control loop [7. To solve these stability problems, both passive and active damping methods are used in the existing literature [8, 9.

There are several passive damping approaches, consisting in the addition of damping resistors that increase the converter power losses . Even though some damping topologies present reduced power losses 10, they introduce other passive components and the filter complexity grows.

Active damping $(\mathrm{AD})$ approaches have been widely explored in previous papers as they can stabilise the system without increasing its power losses. The capacitor voltage feed-forward can effectively damp the filter resonant poles, but it becomes ineffective as the $L C L$ resonance frequency approaches the converter control Nyquist frequency [11. In high power converters, where the switching frequency, and accordingly the sampling frequency, is limited to reduce the power losses, and the resonance frequency is increased to lower the filter size, a different AD strategy is required. A notch filter, inserted in the current control loop and tuned at the resonance frequency, could damp the resonance [12, but it requires an estimation of the effective grid inductance, as it can change depending on the grid at which the VSC is connected and the power injected at the point of common coupling (PCC) 13, 14. Alternatively, a lead-lag controller can be tuned in the current control loop to avoid -180 degree crossings [12] that can lead to instability. However, when the resonance frequency approaches the converter control Nyquist frequency, it is unable to introduce enough phase lead. A suitable option to overcome this limitation is the introduction of additional delays and low-pass filters [15, 16, which are able to stabilise the resonant poles. Nevertheless, they provide a poor damping at the resonant poles, compromising the grid current harmonic content and, therefore, the fulfillment of the grid codes [16. The capacitor current proportional feedback is one of the preferred solutions, equivalent to the implementation of a virtual resistor in parallel with the filter capacitor [17, 18, 19, 20, 21. The main drawback is that it requires additional sensors as this current is not normally measured a in grid-connected VSC. An alternative is using the capacitor voltage, measured for synchronisation purposes, using its derivative to estimate its current and performing an active damping strategy equivalent to the previous one $22,23,24]$.

Both, the capacitor current feedback and the capacitor voltage derivative AD strategies, are based on the emulation of a virtual damping resistor, which becomes a virtual impedance by the effects of the control delays [19, 20]. The real part of the emulated virtual impedance varies with frequency and it can become negative, leading to instability if the resonance frequency is located in the negative region. This issue has been reported in the literature: in [17] the stability region where the capacitor current AD can effectively damp the resonance is limited to $\omega_{s} / 6, \omega_{s}$ being the sampling frequency. The stability region calculated imposes constraints on the $L C L$ filter design: the $L C L$ resonance has to be lower than $\omega_{s} / 6$. This restriction compromises the achievement of an optimised filter in order to meet the grid codes at the lowest price. In [19] they identified the same stability limits, suggesting that the resonance frequency should be limited to be lower than $\omega_{s} / 6$, where the emulated virtual resistance is positive, so that the $\mathrm{AD}$ can stabilise the resonant 
poles. Alternatively, they proposed reducing the computation delay to widen the stability interval. Lastly, [20] proposed an $R C$ virtual damper that modifies the stability region, changing the feedback sign in order to operate at the region where the emulated virtual resistor is negative. In this case the resonance frequency is restricted to a wider interval limited by $\omega_{s} / 5$ and $\omega_{s} / 2$.

In commercial high power converters, as the one further described in this work, the converter side inductance is around 0.1 p.u., the filter capacitor is 0.03 p.u., while the grid side inductance is formed by the transformer leakage inductance, 0.05 p.u, and the effective grid inductance, which is unknown and would modify the filter resonance frequency. The resonance frequency, for the values provided, is bounded within $0.15 \omega_{s}$ and $0.27 \omega_{s}$, as the grid inductance varies from a short circuit ratio (SCR) at the PCC of 1 to 300 . This interval of possible resonance frequencies does not fall within the stability regions identified by the previous papers. To solve this issue, in this article, a different approach is presented. Instead of designing the filter to locate the resonance frequency where the $\mathrm{AD}$ is able to stabilise the resonant poles, the $\mathrm{AD}$ stability region is modified and adapted to be robust and stable within the range of frequencies where the $L C L$ resonant poles can be located for a given power converter design. With this purpose, the delay in the AD path is modified after an analysis of the existing delays in the control loop, considering the filters applied to the measurements. These filters are neglected in the literature, even though they strongly affect the AD stability region and must be introduced to avoid noise amplification by the derivative. Analytical expressions are provided for the adjustment of the delay, which are valid for the capacitor current proportional feedback and the capacitor voltage derivative AD (CVDAD). However, this paper is focused on the latest strategy, as it avoids the use of additional sensors.

The discrete implementation of the derivative close to the control Nyquist frequency is not possible without magnitude and phase distortion. For this reason, a multisampled derivative is used, as done in the passivity based analysis performed in [25. With the multisampled approach the delay is reduced in the feedback path, achieving a wider stability interval in the CVDAD, as it will be shown in this work. Experimental results are provided to validate the proposed AD approach.

\section{System Modelling and Stability Analysis}

\subsection{System Modelling and Control}

Fig. 1 s shows the typical structure of a VSC connected to the grid with an $L C L$ filter. This filter is formed by the converter inductance, $L_{c}$, the filter capacitor, $C_{f}$, the step-up transformer leakage inductance, $L_{\text {transf }}$, and the grid inductance, $L_{g}$ at the PCC. The latter is usually unknown and variable, modifying the filter grid side effective inductance.

The converter side current is usually controlled by means of a proportional-integral (PI) controller in the synchronous reference frame (SRF). For this reason, the $L C L$ filter model will be developed in the $d q$ 


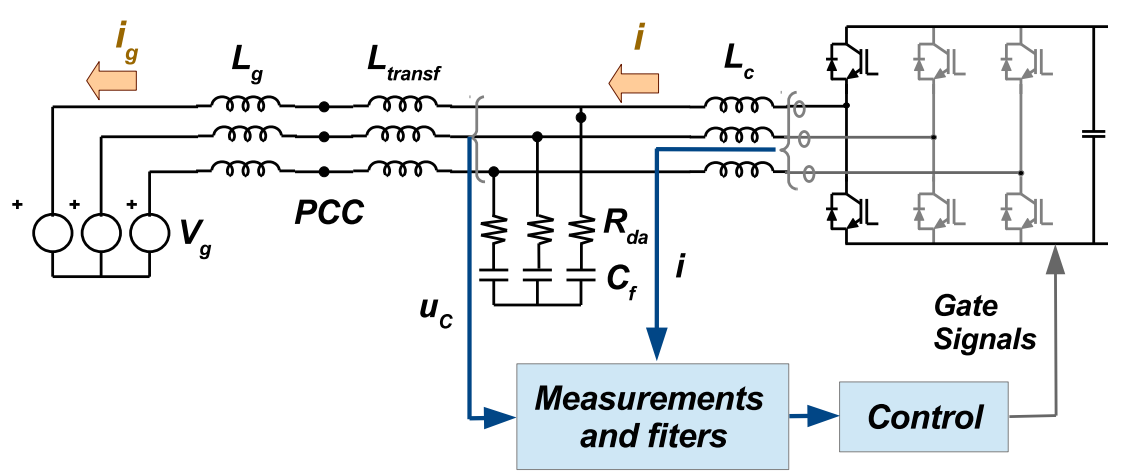

Figure 1: Grid-connected VSC with $L C L$ filter. will be important for the stability analysis performed at the end of this section.

$$
\left[\begin{array}{c}
I_{d}(s) \\
I_{q}(s)
\end{array}\right]=\left[\begin{array}{cc}
G_{1}(s) & G_{2}(s) \\
-G_{2}(s) & G_{1}(s)
\end{array}\right]\left[\begin{array}{c}
V_{\text {conv }_{d}}(s) \\
V_{\text {conv }_{q}}(s)
\end{array}\right]
$$

The expression for $G_{1}(s)$ and $G_{2}(s)$ are given in Eqs. 2 and 3 respectively, reflecting that a sixthorder model is obtained. In these equations $\omega_{r}=1 / \sqrt{C_{f} L}$ is the filter resonance frequency, where $L=$

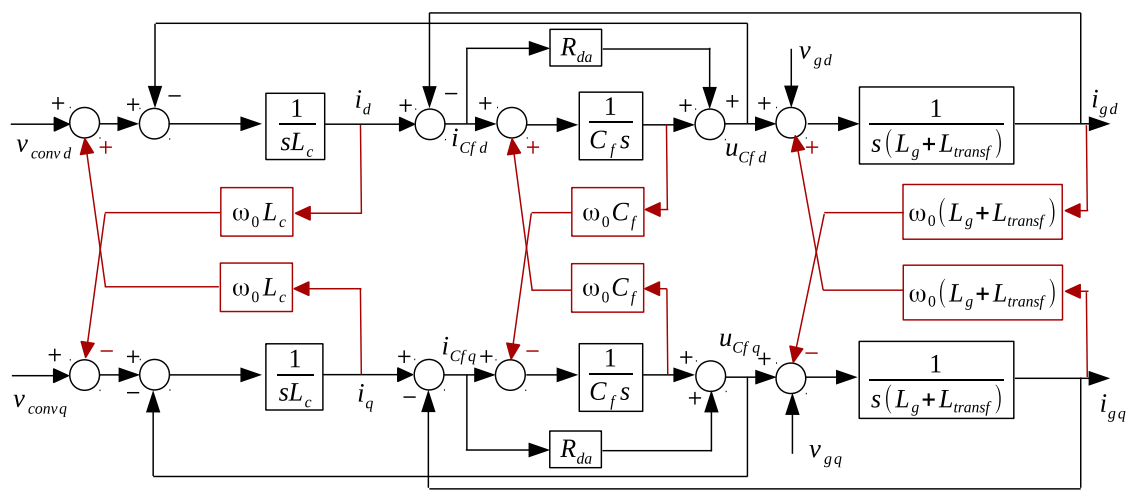

Figure 2: SRF model of the $L C L$ filter. 
inductance, $L_{g}$. The parameters $a$ and $b$ are the ratios between $\omega_{\text {par }} / \omega_{r}$ and $\omega_{0} / \omega_{r}$, respectively, where $\omega_{\text {par }}$ is the parallel resonance frequency between the grid side inductance and the filter capacitor and $\omega_{0}$ is the frequency used for the SRF transformation. Lastly, $Q$ represents the filter quality factor, defined as $R_{d a} / R_{0}$, where $R_{d a}$ is a passive resistor connected in series with the filter capacitor to damp the resonance and $R_{0}$ is $\sqrt{L / C_{f}}$.

In Fig. 3 a schematic of the converter control loop is represented. The capacitor voltage and the converter current measurements are filtered by a first-order low-pass analog filter (LPAF) with a time constant $\tau_{l p}$. These measurements are sampled by the DSP, where the control loops are executed twice per converter switching period. The PLL, receiving the capacitor voltage filtered measurement through a SOGI filter [26], provides the angle for the transformation to the SRF. Each current component is controlled by a PI controller. At the output of the controller, a feed-forward compensation of the capacitor voltage is added through a low-pass digital filter (LPDF). The sum of the controller and feed-forward voltages is saturated to avoid overmodulation, using an anti-windup (AW) for the PI.

The Laplace domain has been chosen to model the system because it is a convenient option to analyze it when the active damping with the multisampled approach is introduced. The Laplace equivalent transfer functions for the digital elements within the control loop, such as the feed-forward LPDF and the PI controller, are used. To properly study the system stability, a representation of all the elements in the same reference frame is required, in this case, the SRF. The LPAF is applied to the real magnitudes of the variables. This filter can be directly expressed in the stationary frame, $\alpha \beta$, without modifying the filter transfer function. In the control loop described in Fig. 3, elements defined in the stationary frame $\alpha \beta$,

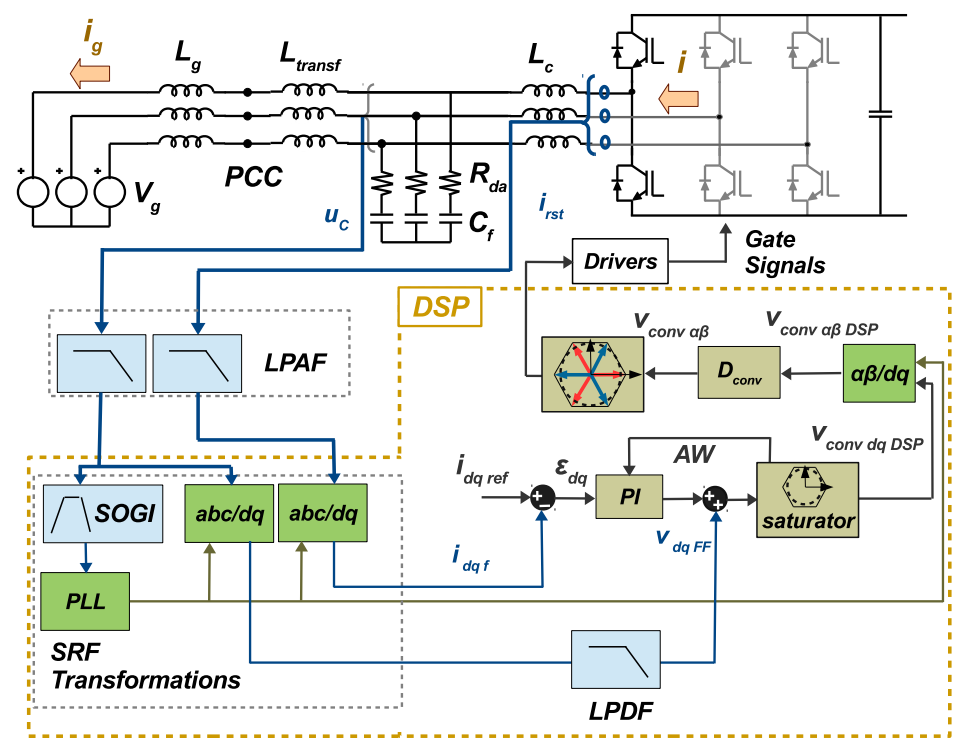

Figure 3: Typical control structure of a grid-connected power converter in the SRF. 
$G_{1}(s)=\frac{1}{L_{c}} \frac{s^{5}+Q \omega_{r}\left(1+a^{2}\right) s^{4}+\omega_{r}^{2}\left(Q^{2} a^{2}+2 b^{2}+\left(1+a^{2}\right)\right) s^{3}+2 Q \omega_{r}^{3}\left(a^{2}+b^{2}\right) s^{2}+\omega_{r}^{4}\left(b^{2}\left(b^{2}+(a Q)^{2}+1-3 a^{2}\right)+b^{2}\right) s+Q \omega_{r}^{5} b^{4}\left(1-a^{2}\right)}{s^{6}+2 Q \omega_{r} s^{5}+\omega_{r}^{2}\left(3 b^{2}+Q^{2}+2\right) s^{4}+2 Q \omega_{r}^{3}\left(2 b^{2}+1\right) s^{3}+\omega_{r}^{4}\left(b^{4}+2(b Q)^{2}+1\right) s^{2}+2 \omega_{r}^{5} b^{2} Q\left(b^{2}+1\right) s+\omega_{r}^{6} b^{2}\left(b^{4}+b^{2}\left(Q^{2}-2\right)+1\right)}$

$G_{2}(s)=\frac{-1}{L_{c}} \frac{\omega_{r} b s^{4}+2 Q b \omega_{r}^{2} a^{2} s^{3}+\omega_{r}^{3} b\left(2 b^{2}+a^{2}\left(3+Q^{2}\right)-1\right) s^{2}+2 Q \omega_{r}^{4} b a^{2}\left(1+b^{2}\right) s+\omega_{r}^{5} b\left(b^{4}+(Q b a)^{2}-b^{2}-(a b)^{2}+a^{2}\right)}{s_{r} s^{5}+\omega_{r}^{2}\left(3 b^{2}+Q^{2}+2\right) s^{4}+2 Q \omega_{r}^{3}\left(2 b^{2}+1\right) s^{3}+\omega_{r}^{4}\left(b^{4}+2(b Q)^{2}+1\right) s^{2}+2 \omega_{r}^{5} b^{2} Q\left(b^{2}+1\right) s+\omega_{r}^{6} b^{2}\left(b^{4}+b^{2}\left(Q^{2}-2\right)+1\right)}$

i.e. $D_{\text {conv }}$ and the transformation of LPAF to $\alpha \beta$, coexist with elements in the SRF, i.e. the PI current controller and the LPDF. $D_{\text {conv }}(s)$ stands for the computation delay in the DSP, and the zero order hold (ZOH). These elements in $\alpha \beta$ are transformed to the SRF using the transformation proposed in [27]. The LPAF applied in the $\alpha \beta$ frame to the measured variables is shown in Eq. 4 for the converter current case, where $L P A F(s)$ is $1 /\left(\tau_{l p} s+1\right)$.

$$
\left[\begin{array}{c}
I_{\alpha, f}(s) \\
I_{\beta, f}(s)
\end{array}\right]=\left[\begin{array}{cc}
L P A F(s) & 0 \\
0 & L P A F(s)
\end{array}\right]\left[\begin{array}{c}
I_{\alpha}(s) \\
I_{\beta}(s)
\end{array}\right]
$$

Eq. 4 is transformed to Eq. 5 its equivalent in the SRF.

$$
\left[\begin{array}{c}
I_{d f}(s) \\
I_{q f}(s)
\end{array}\right]=\left[\begin{array}{cc}
L P A F_{1}(s) & L P A F_{2}(s) \\
-L P A F_{2}(s) & L P A F_{1}(s)
\end{array}\right]\left[\begin{array}{c}
I_{d}(s) \\
I_{q}(s)
\end{array}\right]
$$

where the diagonal terms of the matrix [LPAF] in $d q$ are $L P A F_{1}(s)=L P A F\left(s+j \omega_{0}\right)+L P A F\left(s-j \omega_{0}\right)$ and the anti-diagonal terms are $L P A F_{2}(s)=\left(j L P A F\left(s+j \omega_{0}\right)-j L P A F\left(s-j \omega_{0}\right)\right)$ 27].

The resonance of the $L C L$ filter can be located close to the control Nyquist frequency, so an accurate representation of $D_{\text {conv }}(s)$ is required. The fourth-order approximation calculated in [16] is used and reproduced in Eq. 6.

$$
D_{\text {conv }}(s)=\frac{12 T_{s_{D S P}}^{2} s^{2}-72 T_{s_{D S P}} s+144}{T_{s_{D S P}}^{4} s^{4}+12\left(T_{s_{D S P}}^{3} s^{3}+5 T_{s_{D S P}}^{2} s^{2}+12\left(T_{s_{D S P}} s+1\right)\right)}
$$

where $T_{s_{D S P}}$ is the DSP sampling time. Eq. 7 in the SRF is obtained by applying the same transformation made for the LPAF,

$$
\left[\begin{array}{c}
V_{\text {conv }_{d}}(s) \\
V_{\text {conv }_{q}}(s)
\end{array}\right]=\left[\begin{array}{cc}
D_{1}(s) & D_{2}(s) \\
-D_{2}(s) & D_{1}(s)
\end{array}\right]\left[\begin{array}{c}
V_{\operatorname{conv}_{d} S P}(s) \\
V_{\operatorname{conv}_{q_{D S P}}}(s)
\end{array}\right]
$$

with $D_{1}(s)=D_{\text {conv }}\left(s+j \omega_{0}\right)+D_{\text {conv }}\left(s-j \omega_{0}\right)$ and $D_{2}(s)=j D_{\text {conv }}\left(s+j \omega_{0}\right)-j D_{\text {conv }}\left(s-j \omega_{0}\right)$. 


\subsection{Stability analysis}

The SCR varies depending on the PCC. As the power converter can be connected to strong and weak grids, the SCR can suffer strong variations [28, ranging from SCRs of 1 to 300. The variation in the effective grid inductance seen by the power converter greatly modifies the output filter resonance frequency. To include any possibility in the stability analysis made in this paper, these variations are bounded by the ideal limits of SCR given by 0, obtaining the lowest resonance frequency $\left(F_{r_{l}}\right.$ in Eq. 8$)$ and $\infty$, obtaining the highest resonance frequency $\left(F_{r_{h}}\right.$ in Eq. $9 p$.

$$
\begin{gathered}
F_{r_{l}}=\frac{1}{2 \pi} \sqrt{\frac{1}{C_{f} L_{c}}} \\
F_{r_{h}}=\frac{1}{2 \pi} \sqrt{\frac{L_{\text {trans }}+L_{c}}{C_{f} L_{c} L_{\text {transf }}}}
\end{gathered}
$$

As established in the previous subsection, the model in the SRF is coupled, so the stability must be analysed through the application of MIMO system theory. The open-loop transfer matrix $\left[H_{o l}(s)\right]$, of the system without the proposed $\mathrm{AD}$, is obtained by correlating the filtered converter current, $I_{d q f}(s)$, and the tracking error, $\varepsilon_{d q}(s)$, from Fig. 4 without considering the $\mathrm{AD}$ feedback path. Even if the resulting matrix is too complicated to reproduce here, it retains the symmetry of the matrix presented in Eqs. 1, 5 and 7 and can be represented as in Eq. 10. This symmetry is relevant because the calculation of its eigenvalues is simplified to Eq. 11 and the closed loop stability can be deduced from their frequency response [29].

$$
\begin{gathered}
{\left[\begin{array}{c}
I_{d f}(s) \\
I_{q f}(s)
\end{array}\right]=\left[\begin{array}{cc}
H_{o l 1}(s) & H_{o l 2}(s) \\
-H_{o l 2}(s) & H_{o l 1}(s)
\end{array}\right]\left[\begin{array}{l}
\varepsilon_{d}(s) \\
\varepsilon_{q}(s)
\end{array}\right]} \\
\lambda_{1,2}(s)=H_{o l 1}(s) \pm j H_{o l 2}(s)
\end{gathered}
$$

Once the model of the system has been developed, the stability analysis can be conducted. As an example, the study is going to be performed for the system parameters summarised in Table 1, which are the ones of the experimental set-up. $R_{d a}$ is chosen to be negligible to analyse the worst case from the stability point of view.

Table 1: System parameters

\begin{tabular}{cccc}
\hline \hline \multicolumn{4}{c}{ Table 1: System parameters } \\
\hline$V_{\text {grid }}$ & $690 \mathrm{~V}$ & $L_{\text {conv }}$ & $400 \mu \mathrm{H}$ \\
$S_{\text {rated }}$ & $500 \mathrm{kVA}$ & $L_{\text {transf }}$ & $150 \mu \mathrm{H}$ \\
$F_{S_{D S P}}$ & $5.6 \mathrm{kHz}$ & $C_{f}$ & $100 \mu \mathrm{F}$ \\
$S C R$ & $1-300$ & & \\
\hline \hline
\end{tabular}




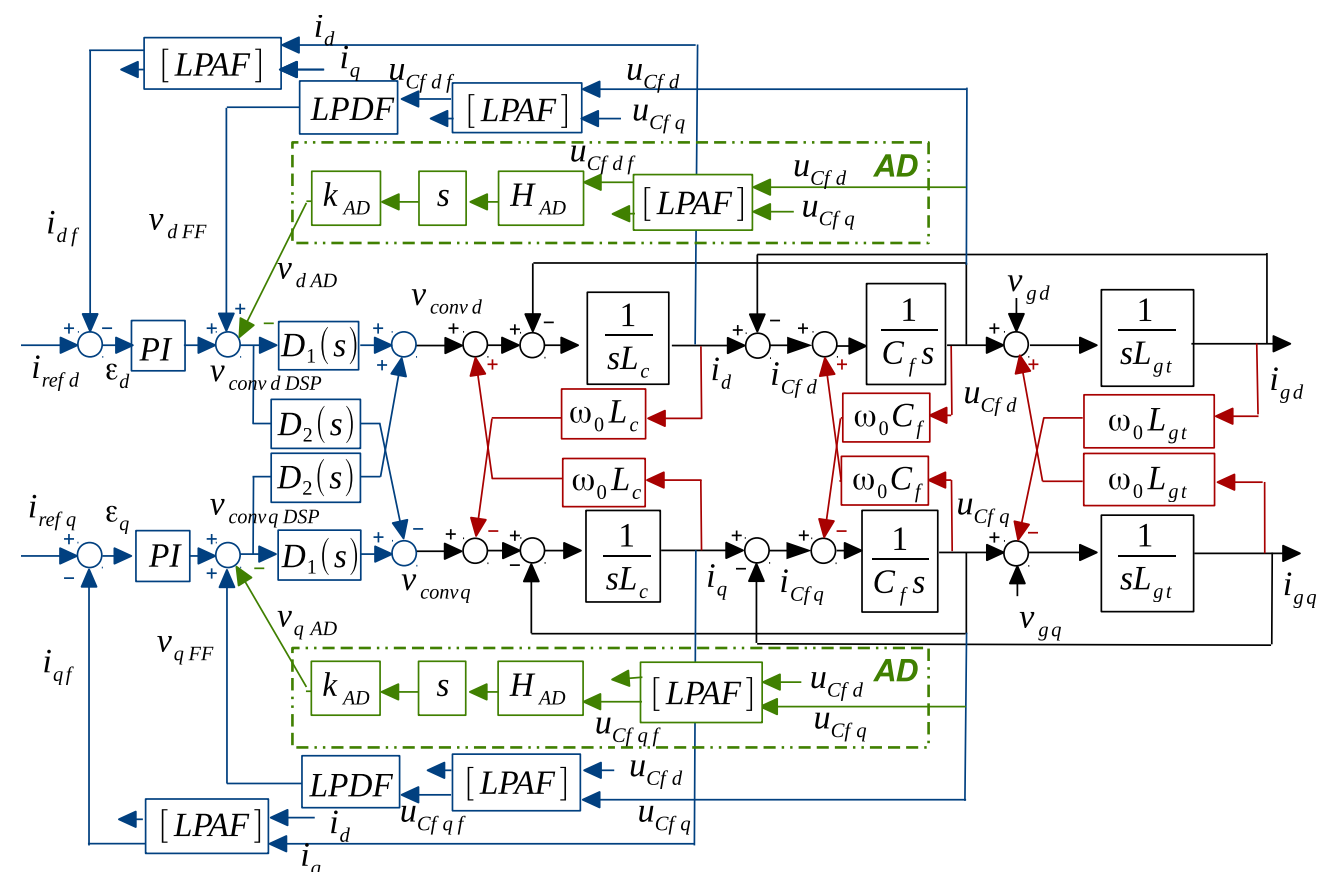

Figure 4: Block diagram representing the control loop in the SRF with the CVDAD.

Due to the existence of complex numbers in the transfer function of the eigenvalues of $\left[H_{o l}(s)\right]$, the Bode plots are not equal at positive and negative frequencies. The frequency response of the eigenvalues, for both positive and negative frequencies, is shown in Fig. 5 for three different SCRs. It can be seen in Fig. 5 (a) and (b) that around the resonance frequency, four -180 degree-crossings occur for each SCR when the magnitude is positive, meaning that the closed loop transfer function will have four unstable poles for any SCR, as the $R_{d a}$ has been neglected. This can be verified when the closed loop poles of the converter current control are represented, Fig. 5 (c), where the four -180 degree-crossings are translated into four unstable poles at the same frequency than the crossings. In a real application, the parasitic resistances of the inductors, capacitors and semiconductors will help stabilise the system for the strongest SCR values. However, for the rest of the cases an active damping method has to be introduced to avoid using additional resistors and consequently increasing the power losses.

\section{Proposed Active Damping Strategy}

\subsection{General description of the active damping strategy}

In Fig. 4 the SRF plant model and control loop, including the AD proposed, are represented. The AD feedback branch, depicted in green, includes the terms of the state-of-the-art solutions: a derivative action multiplied by a constant, $k_{A D}$. However, in this proposal, a new transfer function is added, $H_{A D}$. The main purpose of $H_{A D}$ is adapting the delay in the $\mathrm{AD}$ path to avoid stability problems caused by the variations in 
(a)
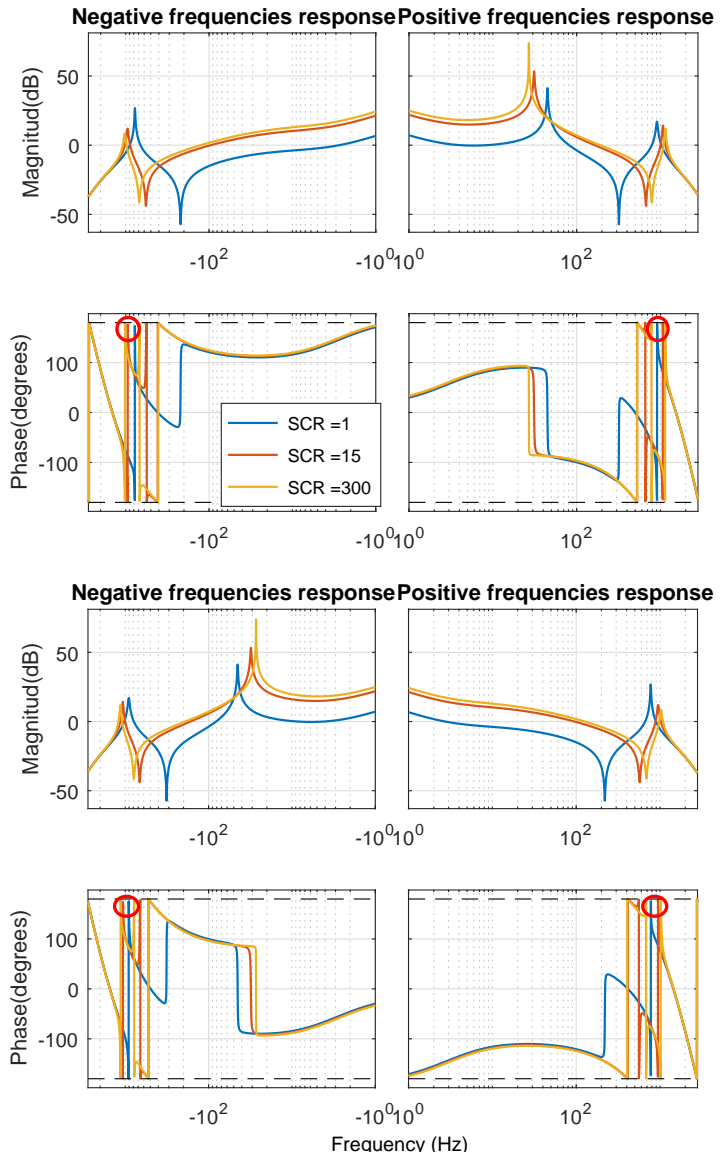

(c)

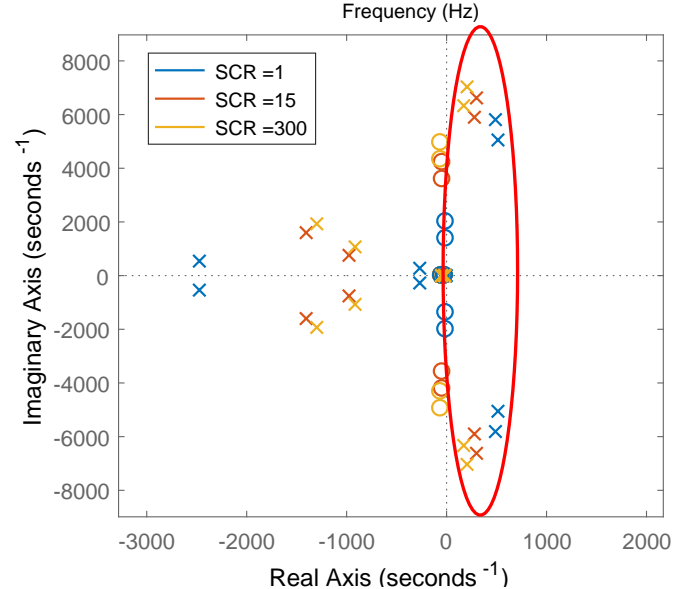

Figure 5: Stability analysis for an SCR of 1 (blue), 15 (red) and 300 (yellow). Bode plot of $\lambda_{1}(s)=H_{\text {ol } 1}(s)+j H_{\text {oll }}(s)($ a), of $\lambda_{2}(s)=H_{o l 1}(s)-j H_{o l 1}(s)$ (b) and pole placement of the current control loop (c).

the sign of the damping action, within all the range of frequencies where the $L C L$ resonance frequency can be located. From Fig. 4 it is clear that the $\mathrm{AD}$ action is affected in the direct path by $D_{1}(s)$ and $L P A F_{1}(s)$ and in the cross path by $D_{2}(s)$ and $L P A F_{2}(s)$. Adjusting $H_{A D}$ with the complete model is complex, for this reason, some simplifications are made. $D_{1}(s)$ always has a magnitude greater than $D_{2}(s)$ and $L P A F_{1}(s)$ 


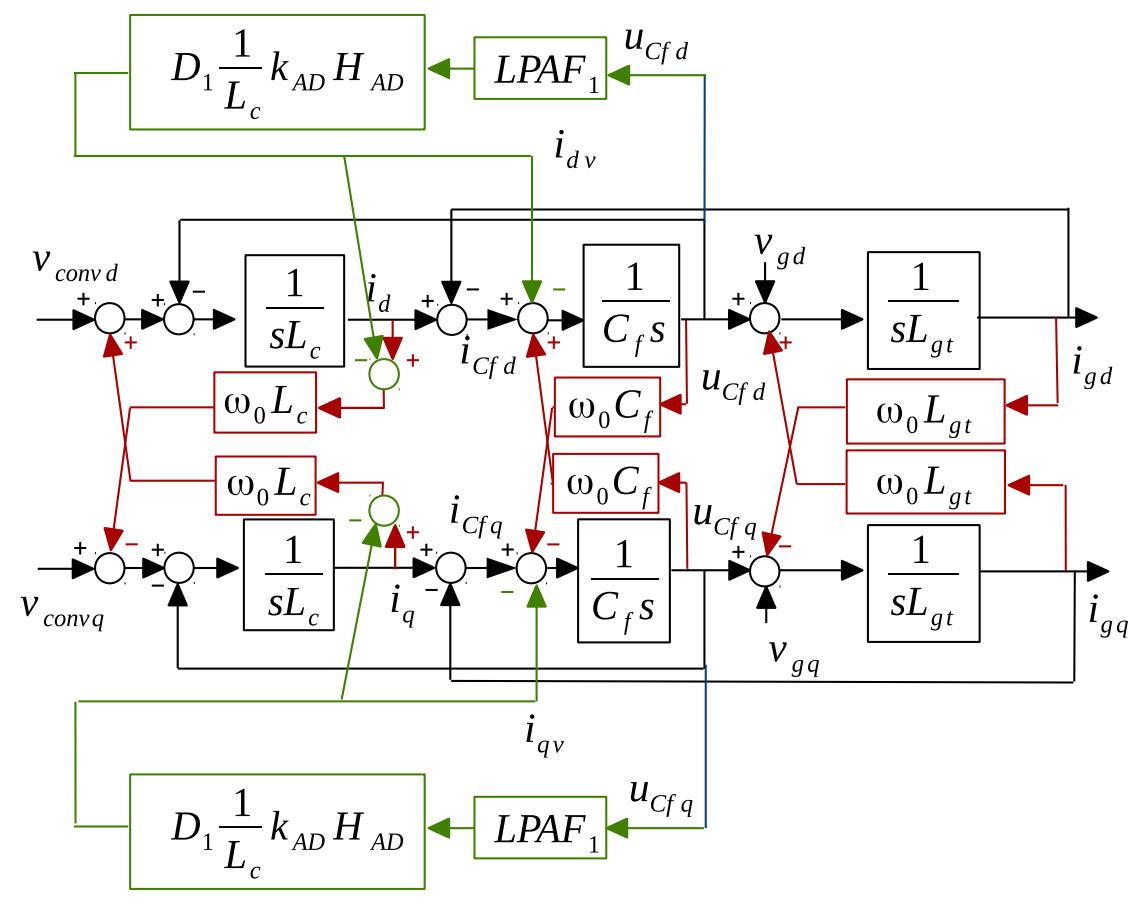

Figure 6: Representation of the CVDAD obtained by simplifying and rearranging the control loop.

is also greater than $\operatorname{LPAF}_{2}(s)$, in this case 14 and 100 times greater respectively, so they can be neglected for the adjustment. By neglecting the cross terms for the AD tuning, the AD control loop diagram can be rearranged as shown in Fig. 6] where only the plant and the AD loop are shown for clarity. With these simplifications it is clear that the $\mathrm{AD}$ feedback is the same in $d$ and $q$ axis, so only the $\mathrm{d}$ axis is studied in the tuning procedure. A virtual impedance can be defined as the ratio of the capacitor voltage in one axis and the virtual current in the same axis and is given by Eq. 12 .

$$
Z_{A D}(s)=\frac{U_{C f d}(s)}{I_{d v}(s)} \approx \frac{L_{c}}{H_{A D}(s) D_{1}(s) k_{A D} L P A F_{1}(s)}
$$

Eq. 12 has the components generally found in the classical AD strategies: a virtual impedance dependent on $L_{c}, k_{A D}, D_{1}$ and $L P A F_{1}$. It is known that the real part of this classical emulated virtual impedance, $\operatorname{Re}\left(Z_{A D}(s)\right)$, or equivalently, its resistive component, changes its value and sign as a function of frequency. A change in the sign of $\operatorname{Re}\left(Z_{A D}(s)\right)$, within the location of the poles at the resonance frequency, causes instability [17, 19, 20. But the additional term $H_{A D}(s)$ that we propose to include in the AD path will allow to modify the delay and guarantee no changes in the sign of $\operatorname{Re}\left(Z_{A D}(s)\right)$ within the range of possible resonance frequencies. Before addressing this key aspect, a proper derivative is required at the $L C L$ resonance frequency, something that becomes complex taking into account the low switching frequencies and the high sampling times of high power converters. 


\subsection{Implementation of the derivative} or phase distortion close to the control Nyquist frequency [23]. The distortion of the derivative depends on its implementation and the sampling time. Both aspects are discussed in this section. Eq. 13 contains the backward Euler discrete implementation of the derivative.

$$
\operatorname{Der}(z)=\frac{1-z^{-1}}{T_{s}}
$$

where $T_{s}$ stands for the sampling time. If this derivative is implemented in the DSP $\left(T_{s}=T_{s_{D S P}}\right)$, which is

where $m$ is a constant that can vary between 0 and 1 . The second proposal is a second order differentiator, given by Eq. 15.

$$
\operatorname{Dif}_{S O}(z)=\frac{2}{T_{s_{D S P}}} \frac{(k+1)\left(2-z^{-1}\right)\left(1-z^{-1}\right)}{2(k+1)+z^{-1}-z^{-2}}
$$

where $k$ is a constant that can vary between 0 and infinity. The parameters $m$ and $k$ modify the frequency response of the derivative close to the control Nyquist frequency. For the representation made in Fig $7 \mathrm{~m}$ has been chosen to be 0.5 and $k$ has been chosen to be 1 , trying to find a compromise between phase flatness within $F_{r_{l}}$ and $F_{r_{h}}$, while keeping the magnitude peak at the control Nyquist frequency at a reduced value.

From Fig. 7 it can be concluded that for the $L C L$ filter parameters under consideration, the derivatives proposed in [23, 30] can perform an accurate derivative within the limits of the $L C L$ resonance frequency. However, in both cases, the error in the phase grows rapidly towards the control Nyquist frequency, $F_{N y}$. In some systems, such as in parallel interleaved power converters, where the $L C L$ resonance frequency is designed to attenuate higher-order harmonics than the first switching harmonic family, the resonance frequency is moved towards the control Nyquist frequency [16], and these differentiators could not be used.

To solve this limitation, the use of a multisampled derivative is proposed. The implementation can be made on an external device, such as a field programmable array (FPGA), or in the same DSP by making 


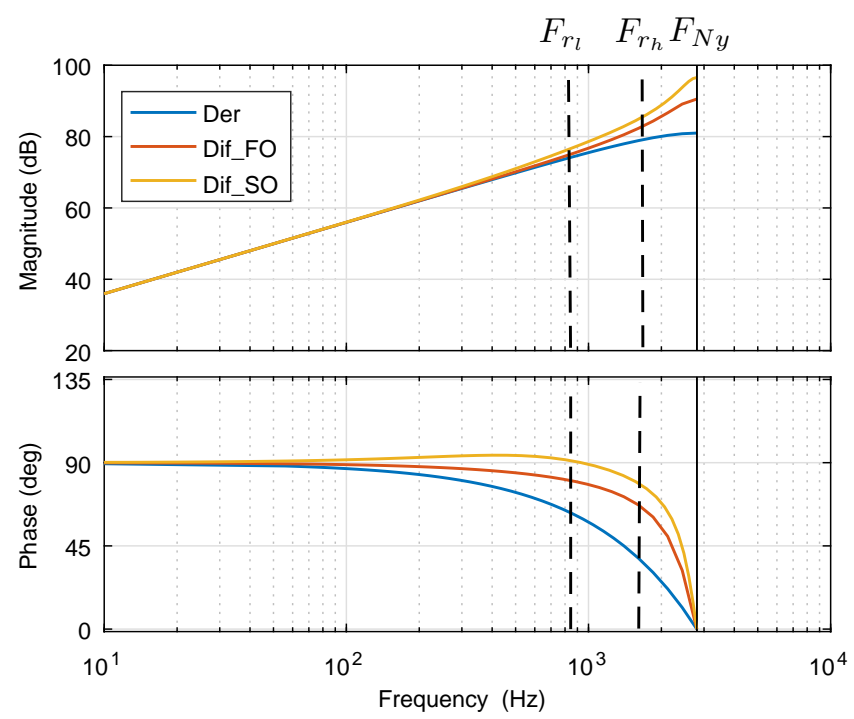

Figure 7: Comparison of the frequency response of the backward Euler derivative and the first- and second-order differentiators proposed in [30] when they are implemented in the DSP.

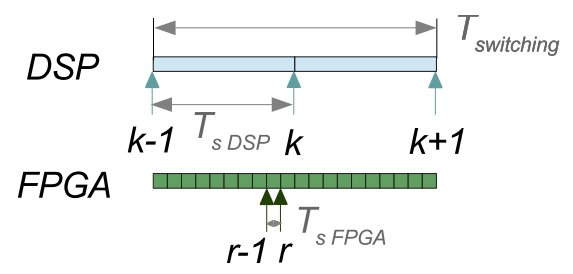

Figure 8: Time diagram illustrating a possible implementation of the multisampled derivative in a FPGA.

use of interrupts. In many applications, the DSP is already complemented by an FPGA [31, running at a faster speed. This is a common approach in high power converters, where the control sampling frequency is limited as a result of the reduced switching frequency. To explain how the multisampled approach can be easily implemented, in Fig. 8 the sample instants of the DSP, $k$, and the FPGA, $r$, are represented. The FPGA only performs the difference between two consecutive samples. At instant $k$ the DSP samples the difference between the FPGA samples $r$ and $r$-1, which are used for the control calculations.

The multisampled derivative is implemented using Eq. 13, and with a high enough ratio of the DSP to the FPGA sampling times, the derivative has almost no phase distortion within the limits of the $L C L$ resonance frequency. This is shown in Fig. 9, where the frequency response of the multisampled backward Euler derivative is plotted for three multisampling ratios, $m r$, of the FPGA sampling frequency to the DSP sampling frequency. If the the multisampling ratio is equal to 10, less than 6 degrees would be lost at the the highest resonance frequency, $F_{R_{h}}$. If the FPGA runs only 4 times faster than the DSP, at the maximum resonance frequency 14 degrees would be lost. If $m r$ is reduced to a minimum of 2 , a higher deformation is obtained in the phase of the derivative and 30 degrees would be lost at $F_{R_{h}}$. For a $m r$ equal to 2 , the 


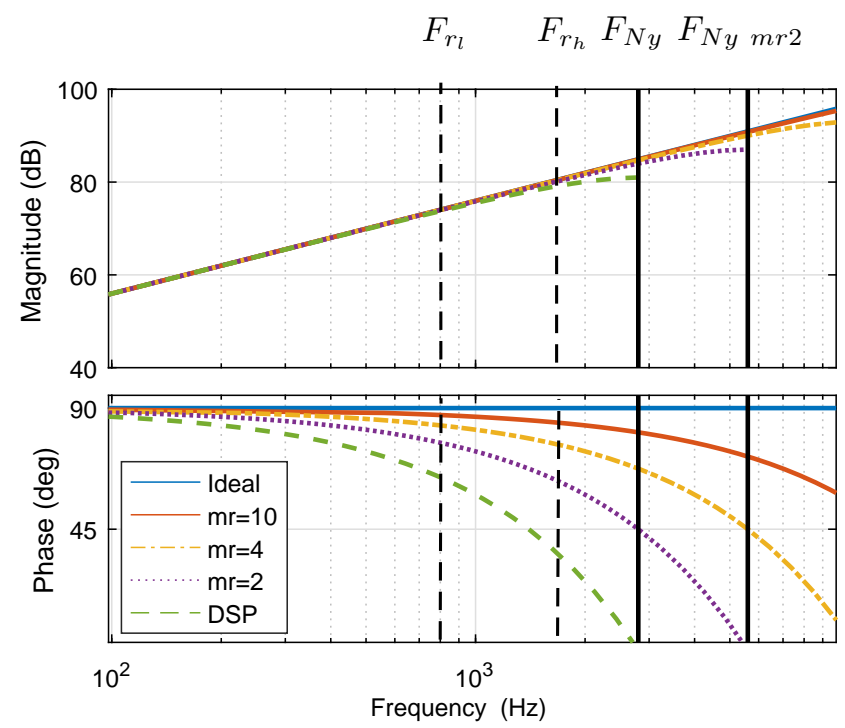

Figure 9: Comparison of the frequency response of the ideal derivative with the backward Euler derivative implemented in the DSP and in an FPGA running 10, 4 and 2 times faster than the DSP.

FPGA Nyquist frequency is denoted in Fig. 9 as $F_{N y} m r 2$.

As a rule of thumb, if the multisampled derivative is 10 times faster than the DSP sampling frequency, an almost ideal derivative is achieved. However, depending on the application and the ratio of the resonance frequency to the control Nyquist frequency, the ratio of the multisampled derivative to the DSP sampling frequency can be reduced.

\subsection{Procedure for the systematic design of $H_{A D}$}

The design of the transfer function applied in the $\mathrm{AD}$ path, $H_{A D}(s)$, is a key aspect to achieve a robust AD. This transfer function, as shown in Eq. 16, includes two terms. The first term is a band pass filter, $B P F(s)$, designed to avoid noise amplification and reduce the required $\mathrm{AD}$ action. The second term is an adjustable delay, $D A D(s)$, included to achieve the desired robustness in the AD strategy.

$$
H_{A D}=B P F(s) D A D(s)
$$

The design of $H_{A D}(s)$ is made in two steps:

- Step 1: Design of the bandpass filter.

$\operatorname{BPF}(s)$ should be able to attenuate both the switching frequency harmonics and the fundamental component (DC in $d q$ ), to avoid noise amplification and to reduce the applied $\mathrm{AD}$ action. However, it should not affect the $\mathrm{AD}$ action inside the range of possible resonance frequencies (from $F_{r_{l}}$ to $F_{r_{h}}$ ), where the $\mathrm{AD}$ is required. As a general rule to achieve these goals, the lower stop-band is set at half the lowest 
$L C L$ resonance frequency, $F_{r_{l}} / 2$, and the highest stop-band is set at $\left(F_{r_{h}}+F_{s w}\right) / 2, F_{s w}$ being the switching frequency. Once both stop-bands have been set, the filter order has to be defined. In this case a second-order filter is used. If a greater attenuation is required, the order of the filter can be increased.

- Step 2: Adjustment of the delay.

If a change in the sign of the emulated virtual resistance occurs within the range of possible $L C L$ resonance frequencies, the system will be unstable for some SCR, as the AD will generate a destabilising action. In Fig. 10 the real part of $Z_{A D}(s)$ is plotted against frequency to illustrate this problem for three different $\mathrm{AD}$ techniques. The CVDAD with the classical derivative, including the $\operatorname{BPF}(s)$ and the $L P A F_{1}(s)$, is able to stabilise the resonant poles for weak grids (lowest resonant frequencies), but it will be unstable if it is connected to strong grids. If the multisampled derivative is used, the delay in the feedback path is reduced and the $\mathrm{AD}$ is able to stabilise the system for strong grids, but not in the weakest cases. However, these changes in the sign of $\operatorname{Re}\left(Z_{A D}(s)\right)$ inside the range of possible resonance frequencies (from $F_{r_{l}}$ to $F_{r_{h}}$ ) can be avoided by modifying the phase, adding an additional delay in the $\mathrm{AD}$ feedback path, $D A D(s)$. $D A D(s)$ is calculated to achieve at the central resonance frequency, $F_{r_{c}}=\left(F_{r_{l}}+F_{r_{h}}\right) / 2$, a pure virtual resistance. As shown in Fig. 10, using the multisampled derivative and the proposed additional delay, $D A D(s)$, the $\mathrm{AD}$ will be able to stabilise the system in the whole range of resonance frequencies, shifting the changes in the sign of $\operatorname{Re}\left(Z_{A D}(s)\right)$ outside the range of possible resonance frequencies to achieve the desired robustness. A negative virtual resistor is emulated, so the AD feedback path sign has to be positive.

As a first step to adjust $D A D(s)$, the phase of $Z_{A D}(s), \varphi\left(Z_{A D}(s)\right)$, has to be characterised and it is given by Eq. 17

$$
\begin{aligned}
\varphi\left(Z_{A D}(s)\right)= & -\varphi\left(D_{1}(s)\right)-\varphi\left(L P A F_{1}(s)\right)-\varphi(B P F(s))- \\
& -\varphi(D A D(s))
\end{aligned}
$$

The additional delay, expressed as the number of sample times, $y$, required to achieve a purely resistive impedance at the central resonance frequency, can be calculated by imposing that, at the central resonance frequency Eq. 17 has to be equal to $\pi$. This equation can be analytically solved to calculate $y$, obtaining Eq. 18

$$
\begin{aligned}
y & =\frac{1}{\omega_{r_{c}} T_{s_{D S P}}}\left(\sum_{i=0}^{n_{z b}} \operatorname{atan}\left(\frac{\omega_{r_{c}}}{z_{B P F_{i}}}\right)-\sum_{i=0}^{n_{p b}} \operatorname{atan}\left(\frac{\omega_{r_{c}}}{p_{B P F_{i}}}\right)+\right. \\
& \left.+\sum_{i=0}^{n_{z l}} \operatorname{atan}\left(\frac{\omega_{r_{c}}}{z_{L P A F_{1_{i}}}}\right)-\sum_{i=0}^{n_{p l}} \operatorname{atan}\left(\frac{\omega_{r_{c}}}{p_{L P A F_{1_{i}}}}\right)+\pi\right)-1.5
\end{aligned}
$$

where $n_{z b}$ and $n_{z b}$ are the number of zeros, $z_{B P F_{i}}$, and poles, $p_{B P F_{i}}$, of $B P F(s)$ respectively. 1.5 stands for the delay of $D_{1}(s)$, and $T_{s_{D S P}}$ is the DSP sampling time. Lastly, $n_{z l}$ is the number of zeros, $z_{L P A F_{1_{i}}}$, and 


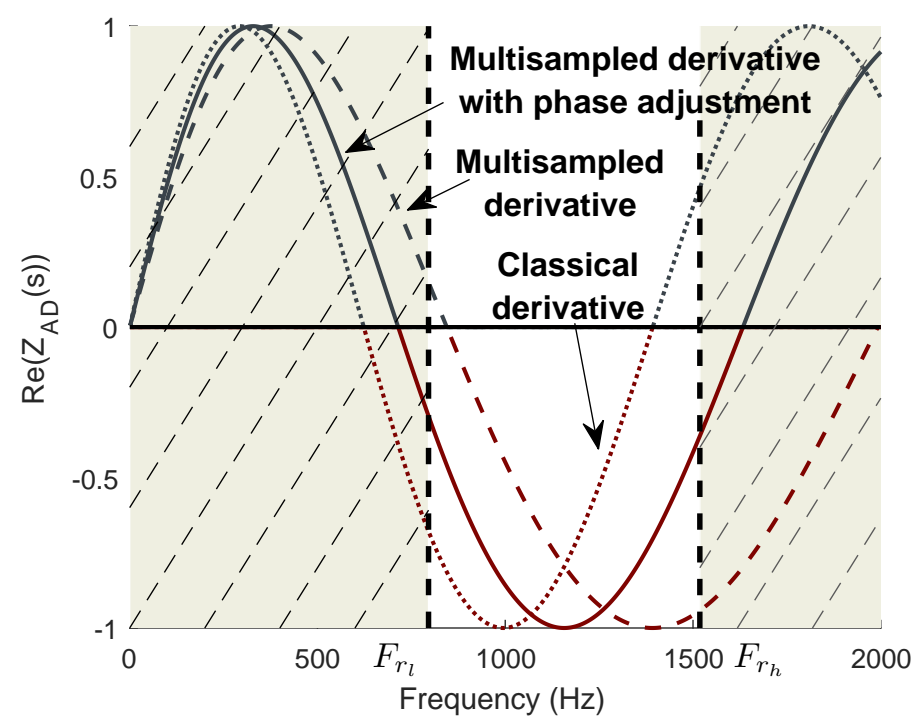

Figure 10: Variation of the virtual impedance real part with frequency for the multisampled derivative, the classical derivative and the proposed approach: the multisampled derivative with phase adjustment.

$n_{p l}$ is the number of poles, $p_{L P A F_{1_{i}}}$, of $L P A F_{1}$. If a first-order low-pass analog filter is considered, when it is expressed in the $\mathrm{SRF}, L P A F_{1}(s)$ has one zero and two poles.

This delay of $y$ sample times has to be programmed in the DSP, where an integer delay can be easily implemented. However, in general, $y$ will be a real number. In this general case, it is decomposed into its integer part, $y_{i}$, and its fractional part, $y_{f}$, which can be implemented in the DSP by means of a linear interpolation 32 , as shown in Eq. 19 .

$$
D A D(z)=\left(\left(1-y_{f}\right)+y_{f} z^{-1}\right) z^{-y_{i}}
$$

It can be seen in Fig 10, represeented for a $m r$ equal to 10, that at the highest resonance frequency the real lost, reamining only 1 degree until the virtual resistance becomes negative. The damping provided is almost negligible and hence, an important ripple could be expected at the $L C L$ resonant poles for high SCRs. For this reason the ratio of the multisampled derivative is such an important aspect.

In this approach, the delay has been adjusted for the widest possible range of resonance frequencies. 
those applications in which the SCR at the PCC is known, by evaluating Eq. 18 at the resonance frequency. In this way, a pure virtual resistor would be emulated at the actual resonance frequency, providing optimal damping at the resonant poles. Once that the delay of the AD path has been properly adjusted, the selection of the emulated virtual resistor would determine the amplitude of the $\mathrm{AD}$ action.

The proper adjustment of the delay guarantees that the phase of the emulated virtual impedance has enough margin before its resistive component changes its sign for any SCR greater than 1 . As a result, the emulated virtual resistance does not need to be modified for the different grid impedances and the robustness is guaranteed.

\subsection{Noise rejection analysis}

It is common in power converters, to sample all the measured variables synchronously, either with symmetrical sampling (once per switching frequency), or with asymmetrical sampling (twice per switching frequency). In this way, aliasing and noise problems are minimised. In the power converter analysed in this work, all the measurements are measured asymmetrically, except for the capacitor voltage measurement used in the CVDAD strategy, as a multisampled derivative is used.

The main source of noise in the measurement of the capacitor voltage is the switching of the power converter. Consequently, the most representative harmonics in the measurement are expected to be located at the switching frequency and its sidebands and all the multiples of the switching frequency and their sidebands. If the multisampled derivative is implemented in an FPGA, the measurement of the capacitor voltage filtered by the LPAF is sampled by the FPGA, and the derivative performed. This derivative will be sampled by the DSP, and the high frequency noise will be translated into low frequency alias at a frequency given by Eq. 20.

$$
F_{\text {alias }}=\left|F_{s_{D S P}}-F_{\text {real }}\right|
$$

where $F_{\text {alias }}$ is the low frequency alias of the real harmonic, with a frequency $F_{\text {real }}$, and where $F_{S_{D S P}}$ is the DSP sampling frequency. The sidebands of the first switching harmonic family appear at $\pm 100 \mathrm{~Hz}$, $\pm 200 \mathrm{~Hz} \ldots$ with the asymmetrical update of the switching orders. When sampled by the DSP, these harmonics are seen as alias with a frequency close to the switching frequency and are attenuated by the band-pass filter. The second switching harmonic family sidebands appear at $\pm 50 \mathrm{~Hz}, \pm 250 \mathrm{~Hz} \ldots$ and are seen by the DSP as alias with a low frequency, which are also attenuated by the bandpass filter previously adjusted. All the switching harmonic families are similarly attenuated and none of the aliases fall within the $\mathrm{AD}$ region. If in a given application, the alias of the switching family falls within the $\mathrm{AD}$ region, an anti-aliasing filter could be implemented in the FPGA. The experimental set-up, in which the CVDAD strategy is tested, will demonstrate that there are no noise problems that compromise the performance and stability of the CVDAD. 


\subsection{Selection of the emulated virtual resistor}

The last step to tune the CVDAD is the selection of the virtual resistor, $R_{d a_{v}}$, defined as the ratio between $L_{c}$ and $k_{A D}$. An initial estimation for $R_{d a_{v}}$ can be found from the expression for the damping of an $L C$ filter with a resistor in parallel with the filter capacitor, Eq. 21. This $R_{d a_{v}}$ would provide the desired damping, $\xi$, when a pure virtual resistance is emulated, however, by the effects of the delays, this can only be achieved at the frequency for which the delay has been adjusted.

$$
R_{d a_{v}}=\frac{Z_{C f}}{2 \xi}
$$

where $Z_{C f}$ is the impedance of the capacitor branch at a given frequency. For the parameters of the case under study, the required $R_{d a_{v}}$ to achieve a damping of 0.25 at the central resonance frequency, where Eq. 21 is valid, results in a value of $2.75 \Omega$. In Fig. 11 the evolution of the resonant poles is plotted for several virtual resistors and an SCR of 10, corresponding to the SCR of the grid at which the experimental set-up is connected and a short circuit ratio that brings the $L C L$ resonance frequency close to the central resonance frequency, $F_{r_{c}}$. It is observed that the proposed $\mathrm{AD}$ is able to stabilise the resonant poles for different virtual resistors, showing robustness faced to variations in this parameter. The greatest damping is achieved for an $R_{d a_{v}}$ close to the initial estimate of $2.75 \Omega$.

\section{Simulation Results}

In the experimental set-up the grid inductance cannot be modified. For this reason, simulations are performed using Matlab Simscape Power Systems in order to validate the proposed AD strategy with delay

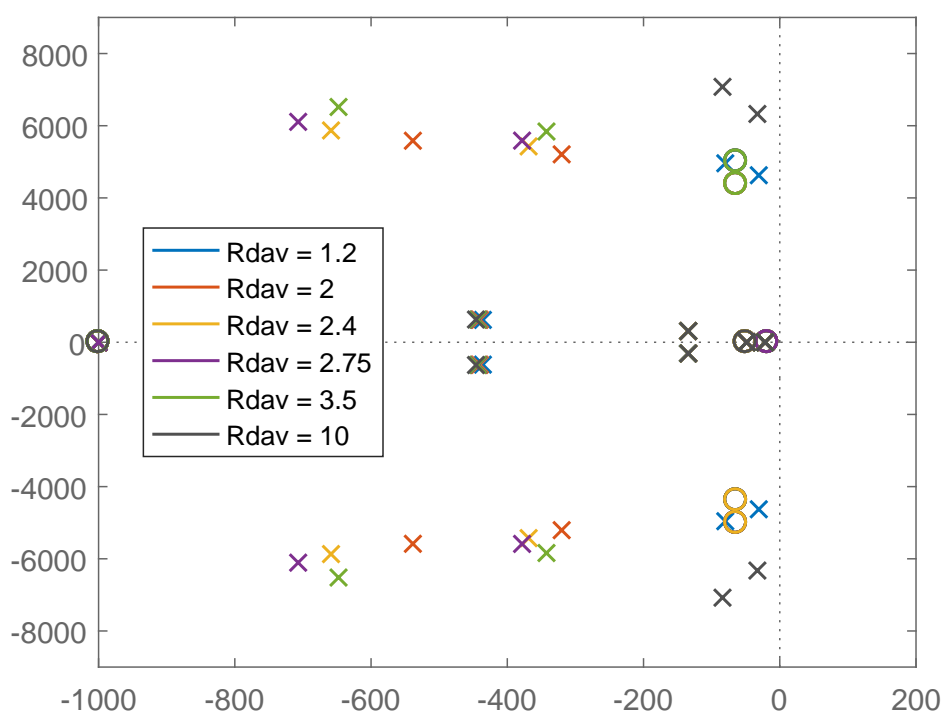

Figure 11: Pole placement of the resonant poles for SCR $=10$ and different virtual resistors. 
(a)

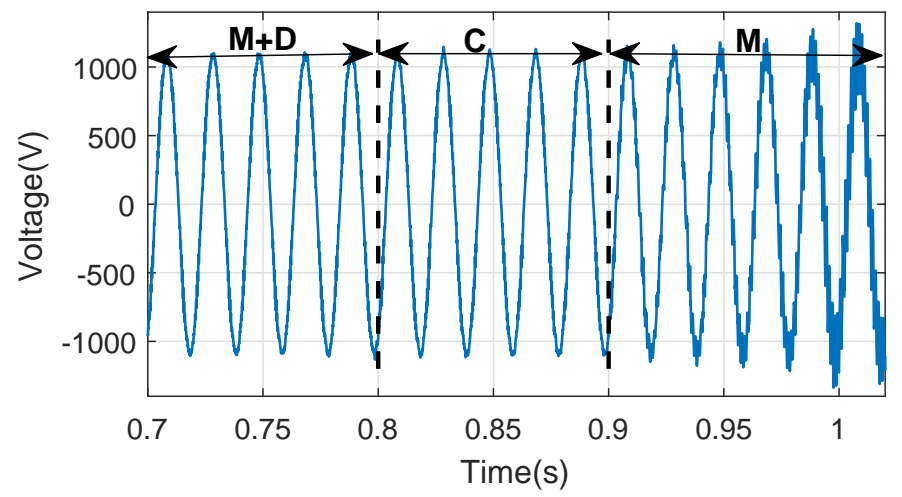

(b)

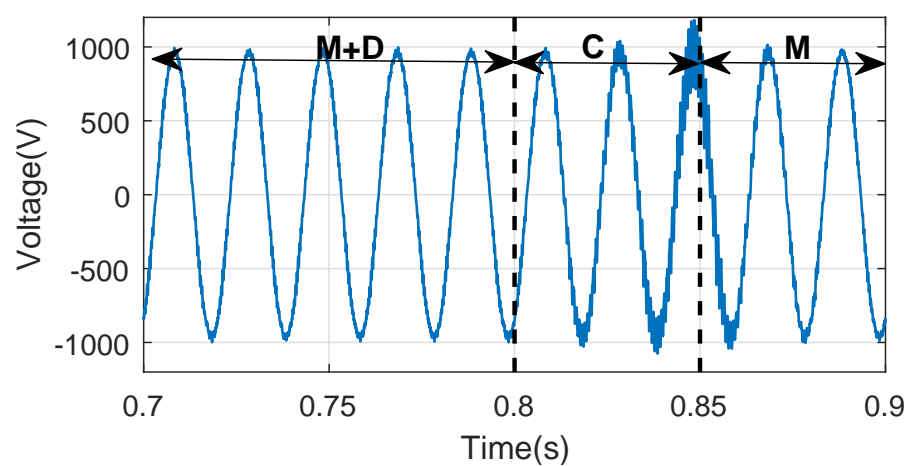

Figure 12: Capacitor line voltage evolution for the three AD strategies and two SCRs: 1.5 (a) and 70 (b).

adjustment.

According to Fig. 10, the multisampled derivative without phase adjustment (M) is able to stabilise the resonant poles for high SCRs (high resonance frequencies), while the classical implementation of the derivative (C) will be able to damp the resonance in weak grids (low resonance frequencies). Only the proposed CVDAD strategy that combines the multisampled derivative with the delay adjustment $(\mathrm{M}+\mathrm{D})$ is able to stabilise the converter for any SCR.

To verify this behaviour two simulations are performed, in which the AD is switched from the multisampled derivative with phase adjustment, to the classical derivative and later to the multisampled derivative without phase adjustment. Two different SCRs are simulated to verify the stability regions obtained in Fig. 10, an SCR of 1.5, corresponding to an $L C L$ resonance frequency of $860 \mathrm{~Hz}$ and an SCR of 70, corresponding to a resonance frequency of $1400 \mathrm{~Hz}$.

If an SCR of 1.5 is considered, both the multisampled with delay adjustment and the classical AD strategy can effectively damp the resonance, as shown in Fig. 12. In contrast, the multisampled strategy without phase adjustment leads the system to instability, and a component at the resonance frequency rapidly grows. As expected from Fig. 10, with the multisampled approach and an SCR of 1.5, the sign of the emulated virtual resistor changes and a destabilising action is applied. In contrast, in a strong grid with 
an SCR of 70, both multisampled strategies, with and without phase adjustment, can damp the system. However, with the classical derivative the system is unstable as the sign of the emulated virtual resistance has changed, confirming again the results in Fig. 10. In this way, the proposed AD is the only strategy able to stabilise the system for any grid considered.

The reduction of the multsampling ratio, $m r$, introduces a phase displacement that cannot be ignored for high SCRs. In Section 3.3 this issue was analyzed based on the theoretical phase displacement obtained in Section 3.2. To validate this theoretical analysis, a simulation has been performed for a SCR of 300, transiently modifying $m r$ from 10 to 4 and later to 2 , the same $m r$ that have been theoretically analyzed. With the original $m r$ of 10 , the system is perfectly stable obtaining a negligible component at the $L C L$ resonance frequency, as shown in Fig. 13. If $m r$ is reduced to 4 , the system becomes closer to instability, as the margin in the emulated virtual resistance is reduced to 17 degrees. The damping of the resonant poles is diminished and an harmonic component at this frequency appears. With a multisampling ratio of only 2 , the margin in the phase of the emulated virtual resistor is only 1 degree and the system is on the verge of instability. An unacceptable harmonic component at the $L C L$ resonant poles frequency is obtained, even though the system is still stable.

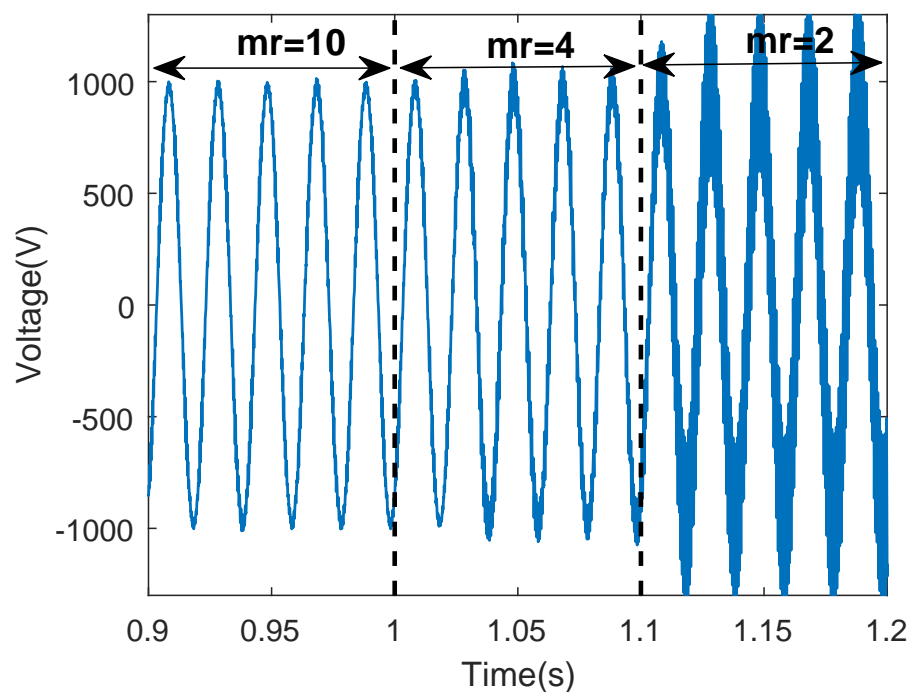

Figure 13: Capacitor line voltage for a SCR of 300 and three different ratios $(m r)$ of the FPGA sampling time to the DSP sampling time.

\section{Experimental Results}

The validity of the approach is tested on the $500 \mathrm{~kW}$ back-to-back power converter shown in Fig. 14 designed for a DFIG wind turbine. Only the grid side converter is used, because the purpose of the test is 
to validate the robust active damping strategy presented. The system parameters are detailed in Table 1 . and it is connected to a grid with an SCR of 10. The DC bus voltage is $1100 \mathrm{~V}$, and the passive resistors, normally used to damp the filter resonance are bypassed. The DSP sampling frequency is set to $5.6 \mathrm{kHz}$ with a converter switching frequency of $2.8 \mathrm{kHz}$ (asymmetrical sampling). An FPGA is used for filtering purposes, running 10 times faster than the DSP and synchronised with it. The AD strategy is implemented following the steps in Section 3. The derivative is implemented in the FPGA and passed to the DSP, where the bandpass digital filter and the adjustment of the delay are applied, emulating a resistor of $2.75 \Omega$.

According to the stability analysis already conducted in Section II, the system without AD is unstable, while if the AD is included it would become stable. This behaviour is validated in the experimental set-up by transiently disabling the $\mathrm{AD}$ action. In Fig. 15 the capacitor line voltages and the converter side current, operating at $40 \%$ of the rated power, are shown as registered by the Yokogawa DL850E. Initially, the AD is activated and the system is stable, but as soon as it is deactivated at instant $t_{1}$, the system becomes unstable and a component at the $L C L$ resonance frequency grows exponentially, verifying the behaviour predicted. When the $\mathrm{AD}$ is activated again after $40 \mathrm{~ms}$, it becomes stable and is able to recover quickly from instability.

The grid current harmonic content with the AD strategy activated is shown in Fig. 16. It can be seen that, when a virtual resistor close the optimal value is emulated, no significant harmonics at the $L C L$ resonance frequency appear, meaning that resonant poles are properly damped, as shown in Fig. 16 (a). Moreover, it can be seen that the grid current has low amplitude harmonics, proving that the AD strategy is robust against noise and aliasing. Nevertheless, if a virtual resistance of $10 \Omega$ is emulated, the resonant poles have a poor damping, and consequently significant harmonics at the resonance frequency appear in

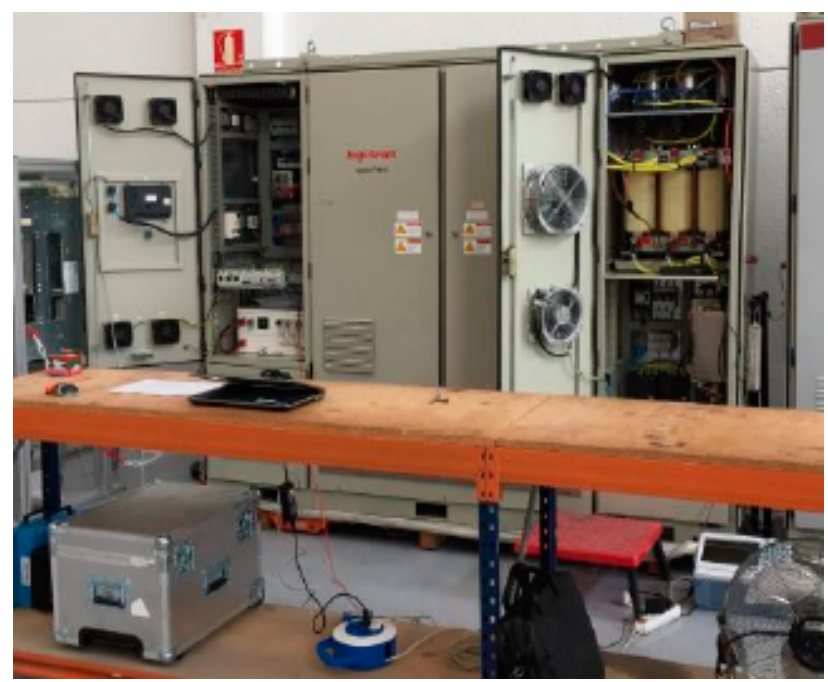

Figure 14: Equipment used validate the AD approach. 


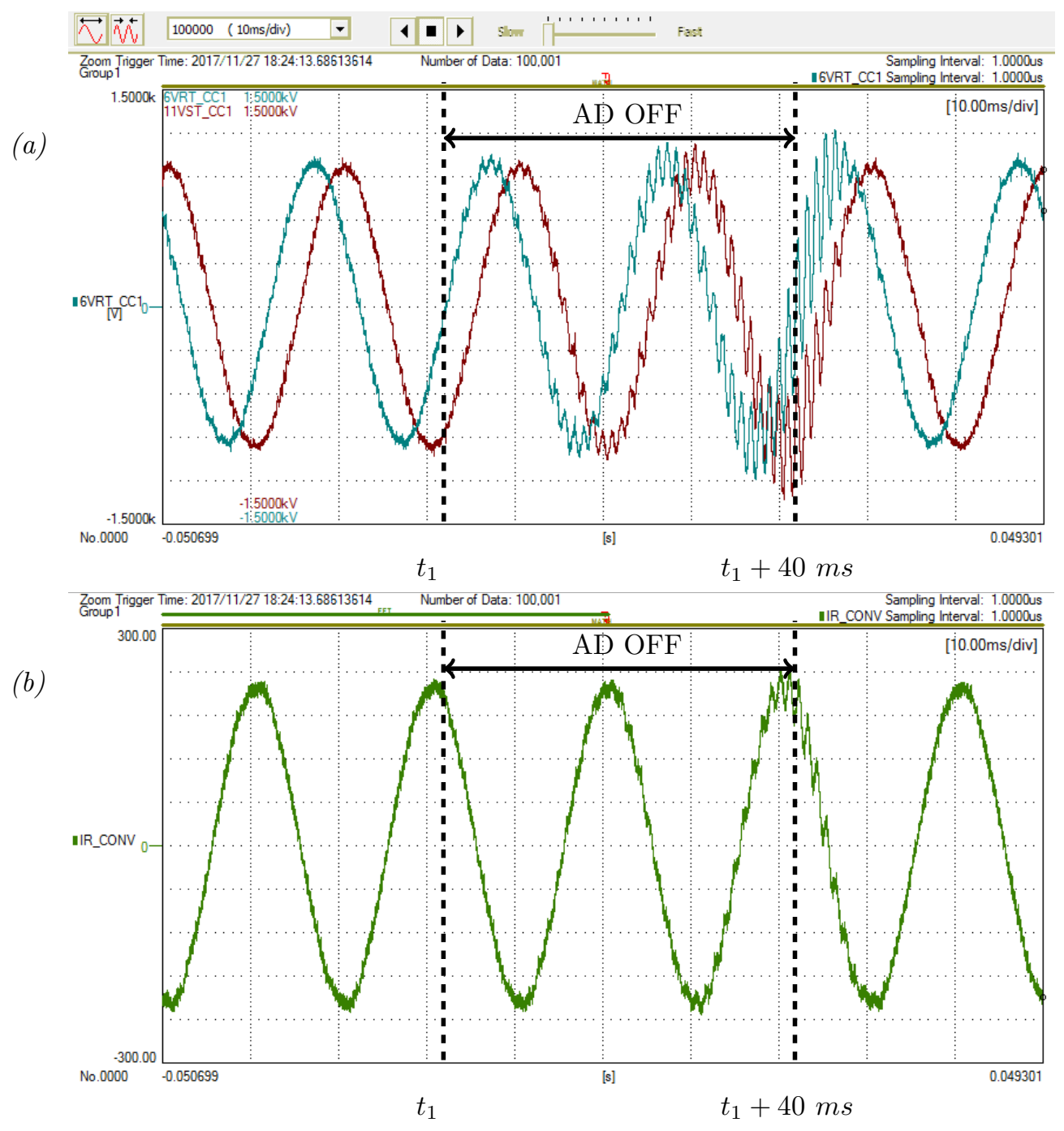

Figure 15: Capacitor line voltage (a) and converter side current (b) evolution when the AD strategy is deactivated for $40 \mathrm{~ms}$. at the resonance frequency. In this way it can be concluded that the detailed modeling approach allows to precisely adjust the AD strategy. 


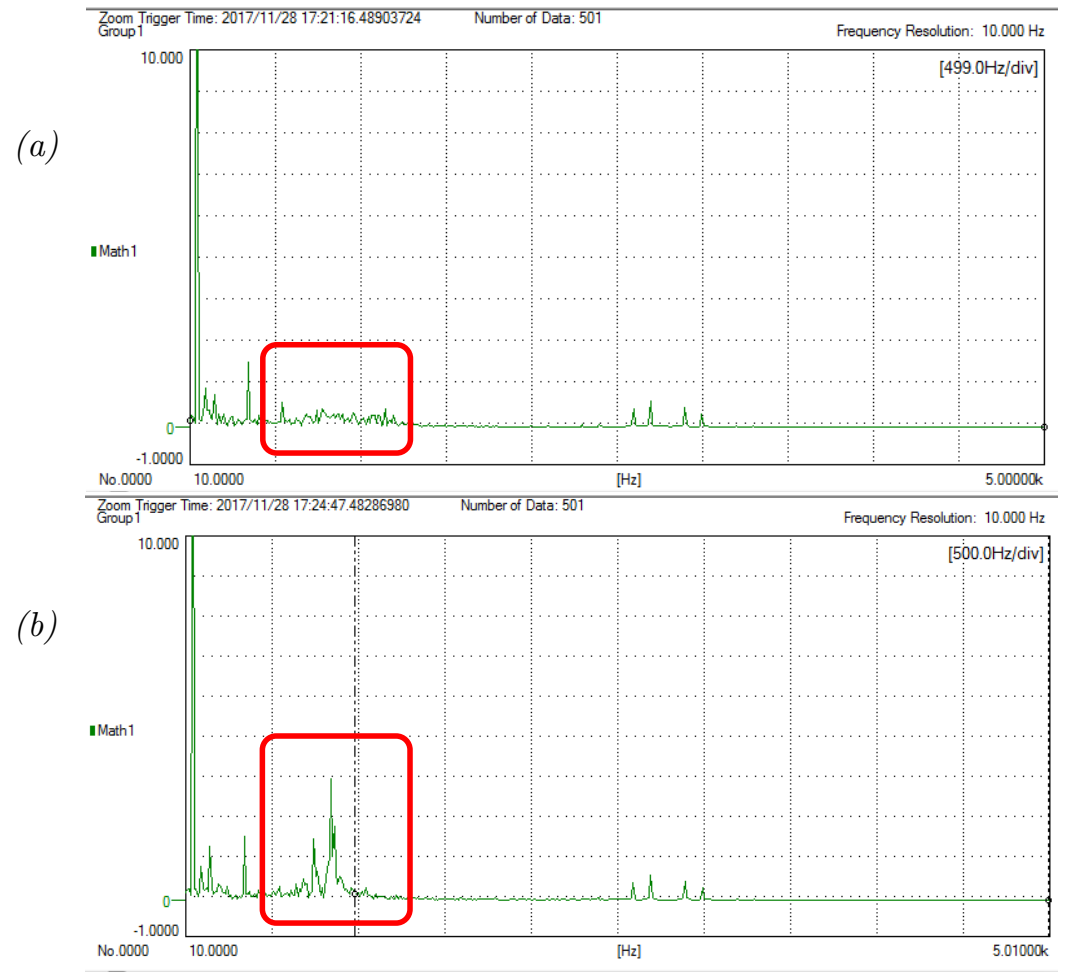

Figure 16: Grid current harmonic content with the AD strategy: emulating a virtual resistor of $2.75 \Omega$ (a) and emulating a virtual resistor of $10 \Omega(\mathrm{b})$.

\section{Conclusion}

In this work a robust active damping strategy based on the capacitor voltage derivative is proposed. With the approach presented, the $\mathrm{AD}$ stability region is adapted to the optimised design of a given $L C L$ filter and all the possible PCCs where the converter can be connected, instead of imposing additional constraints on the $L C L$ design procedure. This goal is achieved by means of an adjustable delay, presenting a systematic procedure to tune this delay in the active damping feedback path by means of an analytical expression that requires reduced information on the control loop. The design procedure and the analytical expression provided to adjust the delay are also applicable to the capacitor current proportional feedback active damping. To overcome the limitations of the derivative close to the control Nyquist frequency, a multisampled derivative is implemented, offering greater robustness against variations in the resonance frequency, as a result of a lower delay. The detailed model developed for the $L C L$ filter and the converter current control loop allows to precisely adjust the active damping strategy. The solution proposed is costless, does not increase the power losses, and is based on the measurements available in grid-connected power converters and the existing digital systems to implement the multisampled derivative. The performance and robustness of the proposed $\mathrm{AD}$ have been tested through experimental and simulation results. 


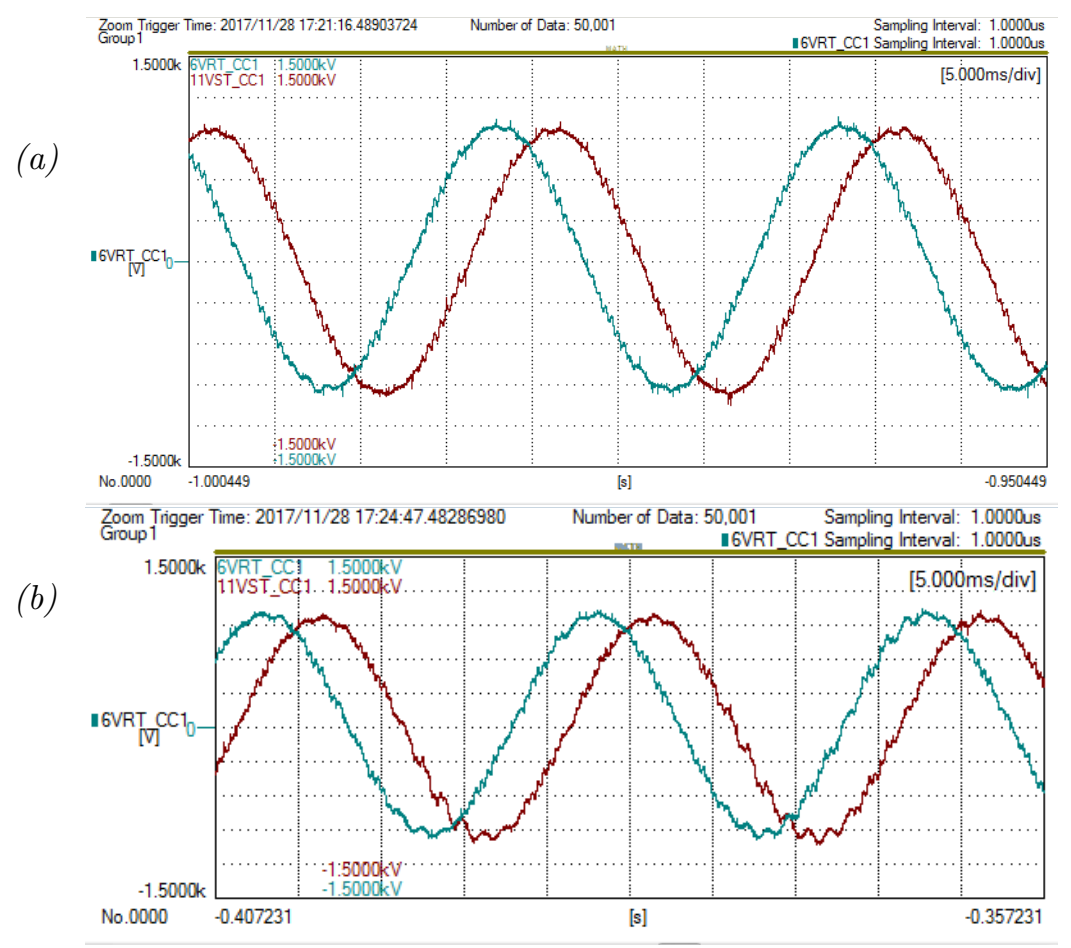

Figure 17: Capacitor line voltages waveforms with the AD strategy: emulating a virtual resistor of $2.75 \Omega$ (a) and emulating a virtual resistor of $10 \Omega(\mathrm{b})$.

\section{Acknowledgements}

This work has been supported by the Spanish State Research Agency (AEI) and FEDER-UE under grant DPI2016-80641-R. This work was partially funded by the Public University of Navarre through a doctoral scholarship. The authors gratefully acknowledge INGETEAM POWER TECHNOLOGY.

\section{References}

[1] F. Blaabjerg, R. Teodorescu, M. Liserre, A. V. Timbus, Overview of control and grid synchronization for distributed power generation systems, IEEE Transactions on industrial electronics 53 (2006) 1398-1409.

[2] J. M. Carrasco, L. G. Franquelo, J. T. Bialasiewicz, E. Galván, R. C. PortilloGuisado, M. M. Prats, J. I. León, N. MorenoAlfonso, Power-electronic systems for the grid integration of renewable energy sources: A survey, IEEE Transactions on industrial electronics 53 (2006) 1002-1016.

[3] Y. P. Kumar, R. Bhimasingu, Electrical machines based dc/ac energy conversion schemes for the improvement of power quality and resiliency in renewable energy microgrids, International Journal of Electrical Power \& Energy Systems 90 (2017) 10-26.

[4] R. Leão, G. Barroso, R. Sampaio, J. Almada, C. Lima, M. Rego, F. Antunes, The future of low voltage networks: Moving from passive to active, International Journal of Electrical Power \& Energy Systems 33 (2011) 1506-1512.

[5] IEEE Application Guide for IEEE Std 1547, IEEE Standard for Interconnecting Distributed Resources With Electric Power Systems, 2009. 
[6] M. Liserre, F. Blaabjerg, S. Hansen, Design and Control of an LCL -Filter-Based Three-Phase Active Rectifier, IEEE Transactions on Industry Applications 41 (2005) 1281-1291.

[7] J. Wang, J. D. Yan, L. Jiang, J. Zou, Delay-dependent stability of single-loop controlled grid-connected inverters with lcl filters, IEEE Transactions on Power Electronics 31 (2016) 743-757.

\section{[15] J. Wang, J. D. Yan, L. Jiang, J. Zou, Delay-dependent stability of
filters, IEEE Transactions on Power Electronics 31 (2016) 743-757}

[16] J. Samanes, E. Gubía, Sensorless active damping strategy for parallel interleaved voltage source power converters with lcl filter, in: Applied Power Electronics Conference and Exposition (APEC), 2017 IEEE, IEEE, 2017, pp. 3632-3639.

[17] S. G. Parker, B. P. McGrath, D. G. Holmes, Regions of active damping control for lcl filters, IEEE Transactions on Industry Applications 50 (2014) 424-432.

[18] J. Dannehl, F. W. Fuchs, S. Hansen, P. B. Thøgersen, Investigation of active damping approaches for PI-based current control of grid-connected pulse width modulation converters with LCL filters, IEEE Transactions on Industry Applications 46 (2010) 1509-1517.

[19] D. Pan, X. Ruan, C. Bao, W. Li, X. Wang, Capacitor-current-feedback active damping with reduced computation delay for improving robustness of lcl-type grid-connected inverter, IEEE Transactions on Power Electronics 29 (2014) 3414-3427.

[20] X. Wang, F. Blaabjerg, P. C. Loh, Virtual rc damping of lcl-filtered voltage source converters with extended selective harmonic compensation, IEEE Transactions on Power Electronics 30 (2015) 4726-4737.

[21] M. B. Saïd-Romdhane, M. Naouar, I. Slama-Belkhodja, E. Monmasson, Time delay consideration for robust capacitorcurrent-inner-loop active damping of lcl-filter-based grid-connected converters, International Journal of Electrical Power \& Energy Systems 95 (2018) 177-187.

[22] M. Liserre, a. Dell'Aquila, F. Blaabjerg, Stability improvements of an LCL-filter based three-phase active rectifier, 2002 IEEE 33rd Annual IEEE Power Electronics Specialists Conference. Proceedings (Cat. No.02CH37289) 3 (2002) 1195-1201.

[23] Z. Xin, P. C. Loh, X. Wang, F. Blaabjerg, Y. Tang, Highly Accurate Derivatives for LCL-Filtered Grid Converter With Capacitor Voltage Active Damping, IEEE Transactions on Power Electronics 31 (2016) 3612-3625.

[24] R. Peña-Alzola, M. Liserre, F. Blaabjerg, R. Sebastián, J. Dannehl, F. W. Fuchs, Systematic design of the lead-lag network method for active damping in lcl-filter based three phase converters, IEEE Transactions on Industrial Informatics 10 (2014) 43-52. 
[25] L. Harnefors, A. G. Yepes, A. Vidal, J. Doval-Gandoy, Passivity-based controller design of grid-connected vscs for prevention of electrical resonance instability, IEEE Transactions on Industrial Electronics 62 (2015) 702-710.

[26] F. Xiao, L. Dong, L. Li, X. Liao, A frequency-fixed sogi-based pll for single-phase grid-connected converters, IEEE Transactions on Power Electronics 32 (2017) 1713-1719.

[27] D. N. Zmood, D. G. Holmes, G. H. Bode, Frequency-domain analysis of three-phase linear current regulators, IEEE Transactions on Industry Applications 37 (2001) 601-610.

[28] A. Etxegarai, P. Eguia, E. Torres, A. Iturregi, V. Valverde, Review of grid connection requirements for generation assets in weak power grids, Renewable and Sustainable Energy Reviews 41 (2015) 1501-1514.

[29] L. Harnefors, Modeling of three-phase dynamic systems using complex transfer functions and transfer matrices, IEEE Transactions on Industrial Electronics 54 (2007) 2239-2248.

[30] D. Pan, X. Ruan, X. Wang, Direct realization of digital differentiators in discrete domain for active damping of lcl-type grid-connected inverter, IEEE Transactions on Power Electronics (2017) 1-1.

[31] E. J. Bueno, A. Hernandez, F. J. Rodriguez, C. Girón, R. Mateos, S. Cobreces, A dsp-and fpga-based industrial control with high-speed communication interfaces for grid converters applied to distributed power generation systems, IEEE transactions on industrial electronics 56 (2009) 654-669.

[32] T. I. Laakso, V. Valimaki, M. Karjalainen, U. K. Laine, Splitting the unit delay [fir/all pass filters design], IEEE Signal Processing Magazine 13 (1996) 30-60. 